\title{
DYNAMIC BEHAVIOUR OF THE ARCH BRIDGE - THE FULL-SCALE TESTING
}

In the paper the full-scale dynamic testing procedure for highway bridges is presented. The procedure is applied on the new highway steel arch bridge in highway R1 at the locality Nitra - Selenec. After a general description of the bridge, some results relative to inspection and the spectral analysis are reported. The testing equipment, methods of evaluating and processing obtained records utilised during the forced vibration of the bridge are described.

\section{Introduction}

Dynamic testing of bridges has been widely used for the appraisal of the correct structural performance in front of its service loading. Several dynamic testing procedures for highway bridges are reported in literature and national codes. The Slovak National Standard STN 736209 "Loading tests of bridges" [1] determines conditions for the performance of dynamic tests for highway bridges. The tests are designed to obtain the basic characteristics of dynamic response:

1. Vibration frequencies and mode shapes for a loaded and unloaded bridge structure calculated from a frequency analysis of the measured data, which are compared with the theoretical amplitudes from a finite-element model of the bridge.

2. The dynamic coefficient (DC) $\delta_{o b s}(x)$ (the dynamic amplification factor) in characteristic locations $(x)$ of the bridge structure for passages of the testing vehicle:

$$
\delta_{\text {obs }}(x)=\frac{S_{\max }(x)}{S(x)}
$$

where:

$S_{\max }(x)$ is the maximum measured dynamic response of the bridge - the deformation or force measured quantity in the place $(x)$,

$S(x)$ is the static (quasi-static) response quantity of the bridge in the place $(x)$ that can be defined as:

- $S(x)=S_{f i l}(x)$ - is the static response obtained by measured filtering the dynamic response,

- $S(x)=S_{\text {craw }}(x)$ - is the maximum static response obtained from the passage of the testing car at a crawl speed over the bridge,

- $S(x)=S_{(\text {thear })}(x)$ - is the maximum theoretical static response obtained from a passage of the testing car over the bridge.
3. The logarithmic decrement coefficient $\vartheta$

$$
\vartheta=\frac{1}{i} \ln \frac{S_{\text {found }}}{S_{i}}
$$

where:

$S_{\text {found }}$ is the primer vibration amplitude of the quantity $S$ of the unloaded bridge,

$S_{i}$ is the $i$-th amplitude counted from the primer amplitude.

The characteristics of the dynamic response of a bridge are measured with various displacement transducers (LVDT - Linear Vertical Displacement Transducers), accelerometers and strain gauges. Hereinbefore dynamic characteristics are the base for the study of the effects of changes in the stiffness of structural elements and the influence on the dynamic response. An alternative to the DC is to carry out complex dynamic analysis of the bridge using the finite element method.

Many sources of dynamic excitations are used to evaluate the dynamic characteristics of bridges:

- Passages of a testing vehicle over the bridge at various speeds as:

- simple smooth passages of the testing vehicle,

- passages over the bridge instrumented by the normalised $(6 \mathrm{~cm}$ high) roughness.

- Use of the normal traffic conditions.

- Impact tests on a characteristic place of the bridge - for example by the impact generated by the back axes of the vehicle from a defined high; or sudden release of static loads.

- Eccentric mass shakers or exciters allowing the excitation of a bridge at predetermined frequencies.

\footnotetext{
* Milan Moravcik ${ }^{1}$, Martin Moravcik ${ }^{2}$

${ }^{1}$ Department of Mechanics, Faculty of Civil Engineering, University of Zilina, E-mail: milan.moravcik@fstav.utc.sk

${ }^{2}$ Department of Structures and Bridges, Faculty of Civil Engineering, University of Zilina,
} 
The paper presents the testing procedure used at the Department of Structural Mechanics, the University of Žilina, applied to the new highway arch bridge in highway R1 at the locality Nitra Selenec, see Fig. 1. The theoretical vibration frequencies and mode shapes for the bridge were calculated using a finite-element model for the bridge [2]. These calculated frequencies were compared with the measured data. It should be noted that the performed analysis can be applied to further important highway bridges.

\section{Description of the bridge}

The tested structure is a two-lane steel arch bridge whose total length is $88.85 \mathrm{~m}$ and its height is $9.59 \mathrm{~m}$. The construction system includes two separated steel arches on which bridge platform is hanged. The platform consists of two main steel girders connected by transverse beams and is stiffed with a reinforced concrete slab $200 \div 300 \mathrm{~mm}$ thick. The platform is hanged on arches by means of 15 hang bars and its ends are fixed into arches by stiff transverse beams. Fig. 1 shows the bridge model and its cross section.

\section{Numerical analysis - Dynamic properties} of the bridge

In order to evaluate the response under specified loading and standards design loads and dynamic characteristics (frequencies, mode shapes) of the bridge, the complete three-dimensional numerical FEM was set up. The analysis was carried out in the linear elastic range using the computer code ANSYS [2]. Tab. 1 gives analytical natural vibration frequencies - the first ten natural frequencies for the bridge loaded by testing vehicle of the type Tatra 815 (22,0 t)

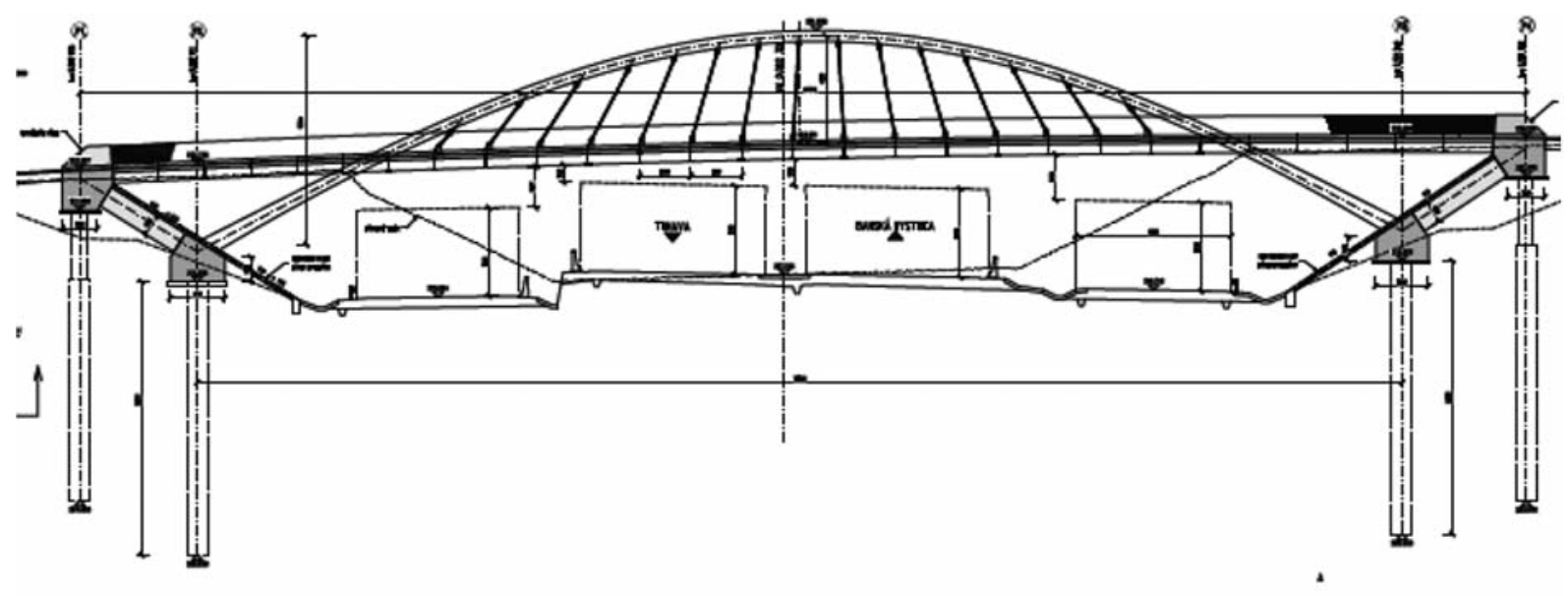

$84.75 \mathrm{~m}$

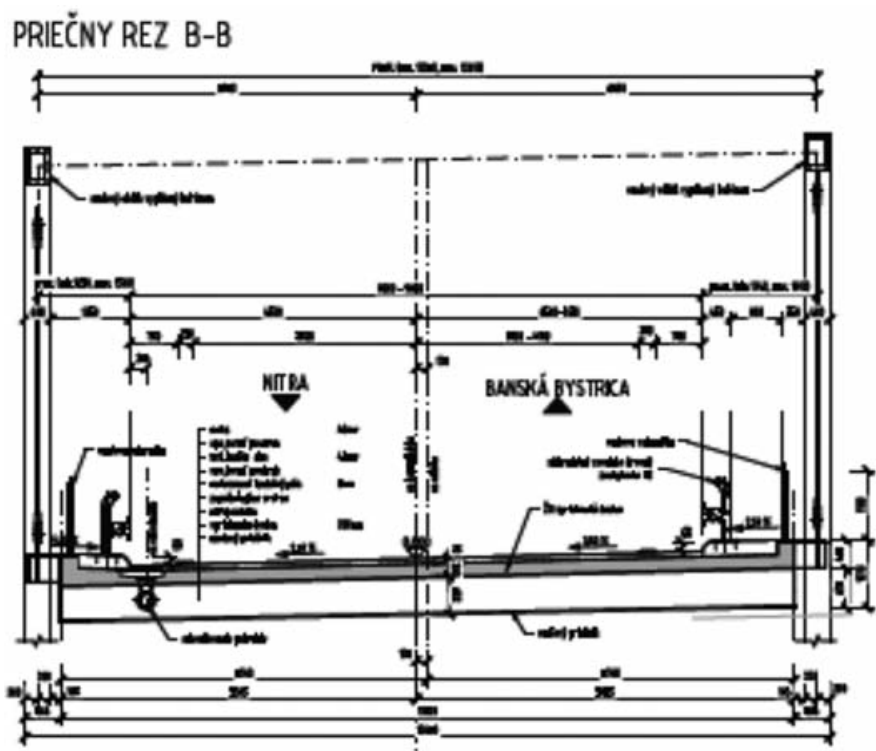

Fig. 1 The bridge model and its cross section - general arrangement. 


\section{COMMNICOIIONS}

The first ten natural vibration frequencies for the bridge loaded by the testing vehicle Tatra 815 (22,0 t)

Tab. 1 set at the middle of the bridge, [2].

\begin{tabular}{|c|c|l|}
\hline \multicolumn{3}{|c|}{ Analytical vibration frequencies } \\
\hline Mode & $\begin{array}{c}\text { Natural frekquency } \\
f_{(i)}[\mathrm{Hz}]\end{array}$ & Shapes of vibration,characteristics of dominant forms of vibration \\
\hline 1 & 0.682 & Horizontal vibration of the left arch (L.A.), the bridge deck does not vibrate. \\
\hline 2 & 0.691 & Horizontal vibration of the right arch (R.A.), the bridge deck does not vibrate \\
\hline 3 & 1.850 & Vibration of arches + bending vibration of the bridge deckin the $1^{\text {st }}$ antisymmetric mode. \\
\hline 4 & 1,883 & Vibration of arches + transverse bending vibration of the bridge deck. \\
\hline 5 & 1.927 & Vibration of arches + bending vibration of the bridge deckin the $1^{\text {st }}$ antisymmetric mode. \\
\hline 6 & 2.040 & Vibration of arches + spatial bending vibration of the bridge deck.+ in the $1^{\text {st }}$ antisymmetric mode. \\
\hline 7 & 2.205 & Bending vibration of the bridge deck in the $1^{\text {st }}$ symmetric mode with the max. amplitudes in the middle of the bridge deck. \\
\hline 8 & 2.403 & $\begin{array}{l}\text { Bending vibration of the bridge deck in the } 1^{\text {st }} \text { antisymmetric mode with the max. amplitudes off the middle the bridge } \\
\text { deck. }\end{array}$ \\
\hline 9 & 2.722 & Transverse bending vibration of the bridge deck in the antisymmetric mode. \\
\hline 10 & 2.948 & Bending vibration of the bridge deck with maximum amplitudes in its ends. \\
\hline
\end{tabular}

at the middle of the bridge. The most important theoretical natural vibration frequencies and corresponding mode shapes are presented in Fig. 2.

The theoretical natural vibration frequencies and corresponding mode shapes were calculated also for the unloaded bridge, the bridge loaded by the vehicle placed in the $1 / 2$ span of the bridge deck, and the bridge loaded by the vehicle placed in the $3 / 4$ span of the bridge deck. These calculate frequencies differ only slightly and are also expected to the dynamic test of the bridge. Frequently occurred natural vibration frequencies and modes in the test are presented in Fig. 2.

- Natural frequency $\boldsymbol{f}_{\mathbf{6}}=\mathbf{2 . 0 4 0} \mathrm{Hz}$ and corresponding mode shape

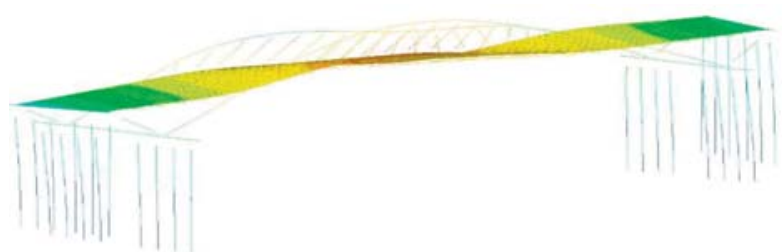

- Natural frequency $f_{7}=\mathbf{2 . 2 0 5} \mathrm{Hz}$ and corresponding mode shape.

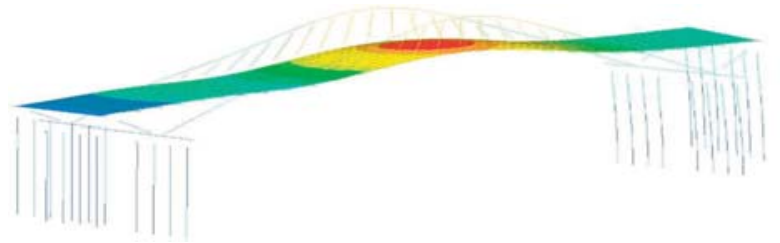

\section{Testing procedure}

During dynamic tests different types of response in the characteristic places $(x)$ of the bridge were measured, see Fig.3:

1. Vertical displacements measured by displacement transducers (LVDTs) of the type BOSH $R_{1} \div R_{2}$ at the $1 / 2$ range of main steel girders, and displacements $R_{3} \div R_{4}$ at the $3 / 4$ range of main steel girders.

2. Strains measured by the strains gauges of the type KISTLER $\mathrm{Tk} 1 \div \mathrm{Tk} 2$ at the $1 / 2$ range of main steel girders, and strains $\mathrm{Tk} 3 \div \mathrm{Tk} 4$ at the $3 / 4$ range of main steel girders.

- Natural frequency $f_{8}=\mathbf{2 . 4 0 3 ~} \mathrm{Hz}$ and corresponding mode shape

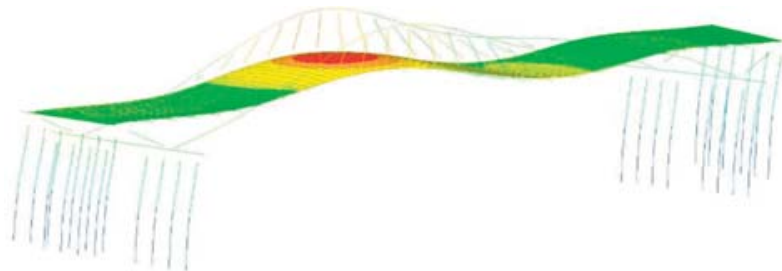

- Natural frequency $f_{9}=\mathbf{2 . 7 2 2 ~ H z}$ and corresponding mode shape.

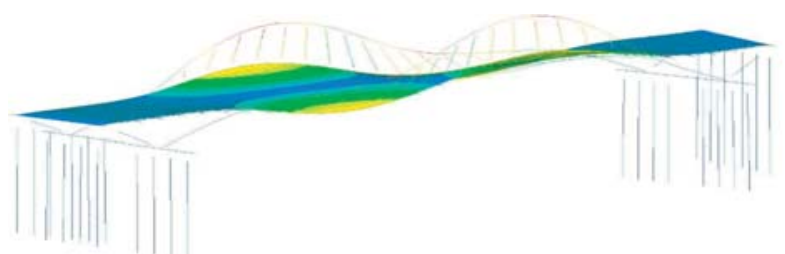

Fig. 2 Frequently occurred natural vibration frequencies $f_{6} \div f_{9}$ in the tests and their corresponding mode shapes for vibration of the bridge deck. 
3. Vertical dynamic components of the displacement $\mathrm{A} 1 \div \mathrm{A} 2$ at the $1 / 2$ range of main steel girders, and components of the displacement $\mathrm{A} 3 \div \mathrm{A} 4$ at the $3 / 4$ range of main steel girders, measured by accelerometers BK 8306 of type BOSH (measured signals transformed direct on the displacements).

4. Vertical dynamic components of the displacement $\mathrm{A} 5 \mathrm{~V} \div \mathrm{A} 6 \mathrm{~V}$ at the $1 / 2$ range of arches (the top section of the arches), and the horizontal dynamic components of displacements $\mathrm{A} 5 \mathrm{H} \div$ $\mathrm{A} 6 \mathrm{H}$ at the $1 / 2$ range of arches (the top section of the arches) measured by accelerometers BK 4508 of type BOSH (measured signals transformed direct on the displacement).

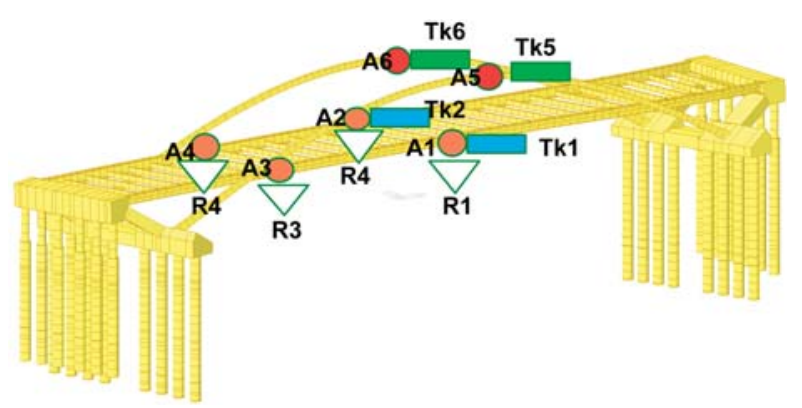

Fig. 3 Location of transducers on the bridge.

Dynamic test were carried out for passages of the testing truck Tatra 815 (the mass $22 \mathrm{t}$ ) at various speeds and positions on the deck:

- Passages of the testing vehicle at the centre line of the bridge:

- symmetric passages of the testing vehicle through the centre of the bridge ("smooth crossing"),

- symmetric passages of the testing vehicle through the centre of the bridge over the normalised ( $6 \mathrm{~cm}$ high) roughness (the normalised plank) placed on the road surface at the centre of the bridge,

- symmetric passages of the testing vehicle through the centre of the bridge over the bridge instrumented by the normalised ( $6 \mathrm{~cm}$ high) roughness placed at the $3 / 4$ of the bridge span.

- eccentric passages of the tested vehicle in the right line.

- Impact tests in the characteristic places $1 / 2$ and $3 / 4$ of the bridge span. The impacts were applied by means of the back axes of the testing vehicle from a defined height $8 \mathrm{~cm}$ on the bridge deck.

The runs of the testing truck were done from the crawling speed to obtain the quasi-static loading response to the speed of $50 \mathrm{~km} / \mathrm{h}$. During the passages the dynamic response were synchronously recorded by all transducers on the computer-controlled data acquisition system DIAEM [3]. The block diagram of the measured and evaluation line is in Fig. 4.

\section{Analysis of measured data}

As provided in Chapter 1, dynamic tests are designed to obtain the basic characteristics of the bridge dynamic response. Obtained time measurement data were analysed in the time and the frequency

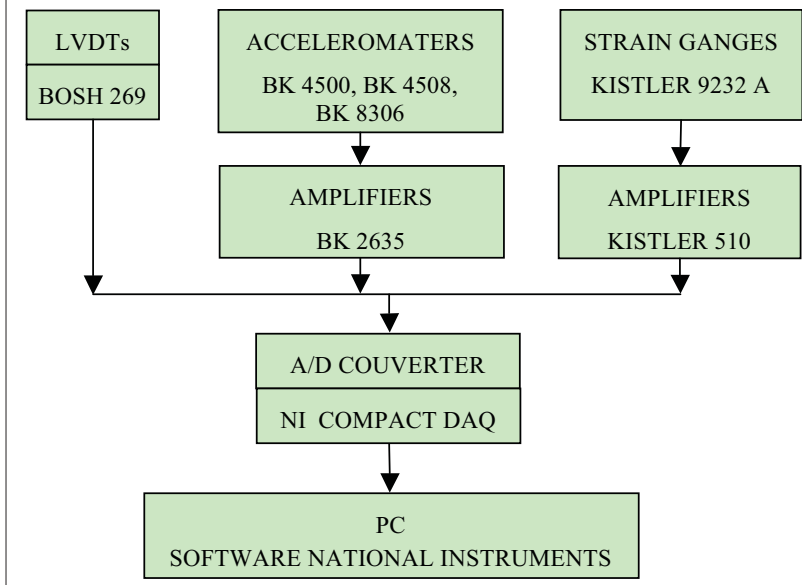

Fig. 4 The block diagram of the measured and evaluation line.

range by the graphical programming environment software LabVIEW.

\subsection{The observed dynamic coefficient (DC) $\delta_{\text {obs }}(x)$}

The DC $\delta_{\text {obs }}(x)$ at a place $(x)$ can be evaluated under controlled passage conditions - passages of the testing truck at different speeds over the bridge. The DC is defined by the relationship (1). The DC at a characteristic place $(x)$ can be evaluated:

- directly from the vertical displacement time history $w_{d y n}(x, t)$ (transducers $\mathrm{R} 1 \div \mathrm{R} 4)$ - this DC is specified as ${ }^{(w)} \delta_{\text {obs }}(x)$,

- directly from the time histories of strain $\varepsilon_{d y n}(x, t)$ - the DC is specified as ${ }^{(\varepsilon)} \delta_{\text {obs }}(x)$,

- combined method, using the static bridge response $w_{s t}(x)$ obtained from tests or from the theoretical solution of a time history of displacement, and a dynamic increment of displacement $\Delta w_{d y n}(x, t)$ measured by accelerometers. The time history of dynamic deflection $w_{d y n}(x, t)$ at a characteristic place $(x)$ then can be expressed:

$$
w_{d y n}(x, t)=w_{s t}(x)+\Delta w_{d y n}(x, t) \rightarrow{ }^{(\Delta w)} \delta_{d y n}(x) .
$$

The corresponding DC is indicated as ${ }^{(\Delta w)} \delta_{o b s}(x)$.

The reference value of the static quantity $S(x) \rightarrow$ the static (quasi-static) response quantity (a displacement $w_{s t}(x)$, or the strain $\varepsilon(x)$ ) of the bridge in the place ( $x$ ) was defined in the relationship (1). Examples evaluated dynamic coefficients ${ }^{(w)} \delta_{o b s}(x),{ }^{(\varepsilon)} \delta_{o b s}(x)$ and ${ }^{(\Delta w)} \delta_{o b s}(x)$ are presented in Figs. $5 \div 7$.

- Dynamic coefficient ${ }^{(w)} \delta_{o b s}(3 / 4)$ - The smooth symmetric passages of the testing vehicle.

File No. 3.6-R3: Transducer R3 placed at the $3 / 4$ span at the left main steel girder (L.G.) of the deck, Fig. 5. The vehicle speed $c=12.4 \mathrm{~m} / \mathrm{s}=44.6 \mathrm{~km} / \mathrm{h}$, the time of passing $t_{p}=7.0 \mathrm{~s}$. 
Dynamic coefficient:

${ }^{(w)} \delta_{o b s}(3 / 4)=\frac{w_{\max }(3 / 4)}{w_{s t}(3 / 4)}=\frac{3.703}{3.423}=1.08$

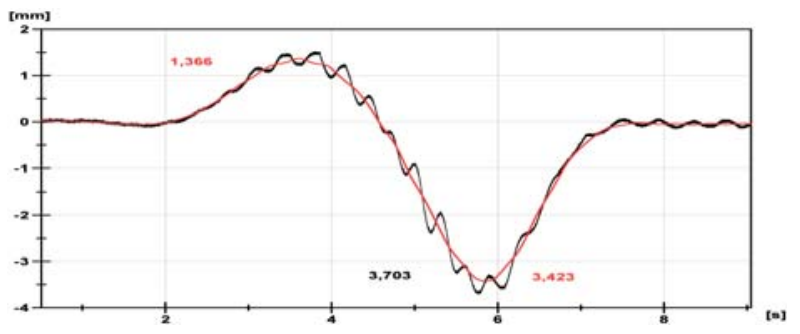

Fig. 5 Time history of the vertical displacement $w_{d y n}(4 / 5, t)$ and corresponding $D C^{(w)} \delta_{\text {obs }}(3 / 4)$ obtained directly from the vertical displacement time history $w_{d y n}(4 / 5, t)$.

- Dynamic coefficient ${ }^{(w)} \delta_{o b s}{ }^{*}(3 / 4)$ - The symmetric passage over the normalised roughness set at $x=1 / 2$.

File No. 4.6-R3: Transducer R3(3/4) placed at the $3 / 4$ span (L.G.) of the deck. The vehicle speed $c=41,7 \mathrm{~km} / \mathrm{h}$, the passage time $t_{p}=7,5$ s Fig. $6 \mathrm{a}, \mathrm{b}$.

Dynamic coefficient:

$\delta_{o b s}{ }^{*}(l=3 / 4)=\frac{w_{\max }(3 / 4)}{w_{s t}(3 / 4)}=\frac{5.025}{3.485}=1.44$.

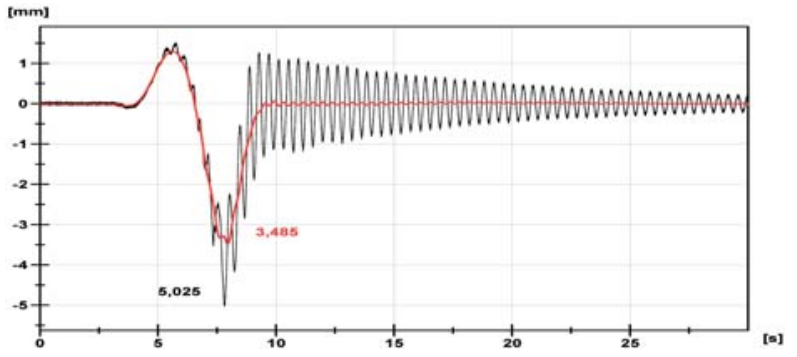

Fig. 6a Time history of the vertical displacement $w_{d y n}{ }^{*}(4 / 5, t)$ and corresponding $D C^{(w)} \delta_{\text {obs }}(3 / 4)$ obtained from the passage over the normalised roughness.

- Damping - Logarithmic decrement of damping $\vartheta$ Logarithmic decrement of damping $\vartheta$ is evaluated from the relationship (2), Fig. 6a:

$$
\vartheta=\frac{1}{20} \ln \frac{w_{1}}{w_{20}}=\frac{1}{20} \ln \frac{1.12}{0.50}=0.0403
$$

- Frequency composition of the vertical displacement $w(3 / 4, t)$, Fig. $6 b$.

Comparison of forced vertical displacements in Figs. 5 and 6 gives the comparison of the smooth symmetric passage and the

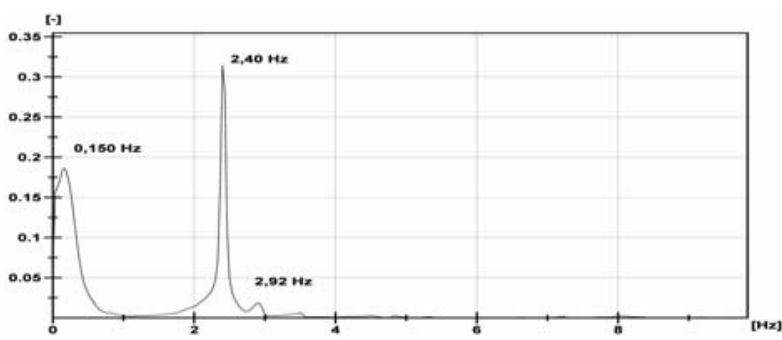

Fig. $6 b$ Frequency composition of the forced vertical displacement $w_{d y n}{ }^{*}(3 / 4, t)$ corresponding Fig. $6 a$.

passage through the normalised roughness on the dynamic coefficient ${ }^{(w)} \delta_{o b s}{ }^{*}(3 / 4)$, and the free vertical vibration of unloaded bridge after the passage of the testing vehicle. Evaluating the logarithmic decrement of damping $\vartheta$ from Fig. 6a gives its typical small value corresponding to steel bridges.

The dominant frequency of the forced vertical vibration $w_{d y n}{ }^{*}(3 / 4, t)$ for the passage of vehicle for the roughness is $\boldsymbol{f}=\mathbf{2 . 4 0}$. This frequency well corresponds to the bending vibration of the bridge deck in the 1st. antisymmetric mode (the natural frequency $f_{8}=2.403 \mathrm{~Hz}$ ) with maximum amplitudes of the vibration which correspond to the position of the testing car out of the middle of the bridge, see Fig. 6a. The other forced frequency $f=2.92 \mathrm{~Hz}$ corresponds to the natural frequency $f_{\mathbf{1 0}}=\mathbf{2 . 9 4 8 ~} \mathbf{H z}$, but this has the minority effect on the bridge vibration.

- Dynamic coefficient - combined method ${ }^{(\Delta w)} \delta_{o b s}{ }^{*}(1 / 2)$ - The symmetric passage over the normalised roughness in the centre of bridge.

File No. 3.3 comb $-\boldsymbol{R} \boldsymbol{2}_{\text {qst }} \mid \boldsymbol{A 2}$ : Transducer $R 2_{\text {qst }}(1 / 2)$ placed at the $1 / 2$ span (R.G.) of the deck, accelerometer $A 2(1 / 2)$ placed at the $1 / 2$ span (R.G) of the deck. The vehicle speed $c=25.9 \mathrm{~km} / \mathrm{h}$, the passage time $t_{p}=12.0 \mathrm{~s}$, Fig. 7a, b.

Dynamic coefficient:

$$
{ }^{(\Delta w)} \delta_{o b s}{ }^{*}(1 / 2)=\frac{w_{\max }(1 / 2)}{w_{s t}(1 / 2)}=\frac{4.795}{3.998}=1.20 .
$$

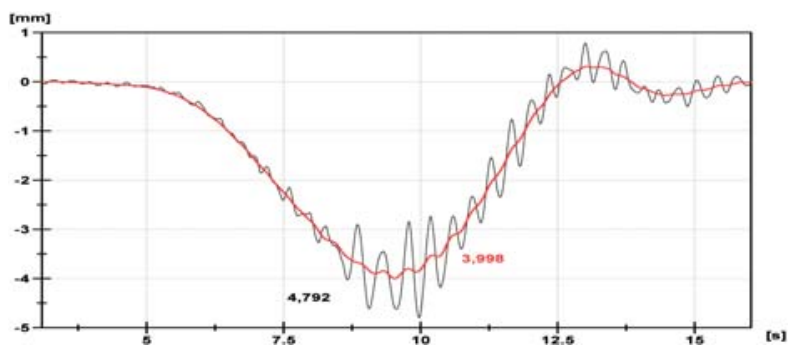

Fig. 7 a Time history of the vertical displacement $w_{d v n}{ }^{*}(1 / 2, t)$ - combined method (the transducer $R 2_{q s t}(1 / 2)$ + accelerometer $A 2(1 / 2))$. 


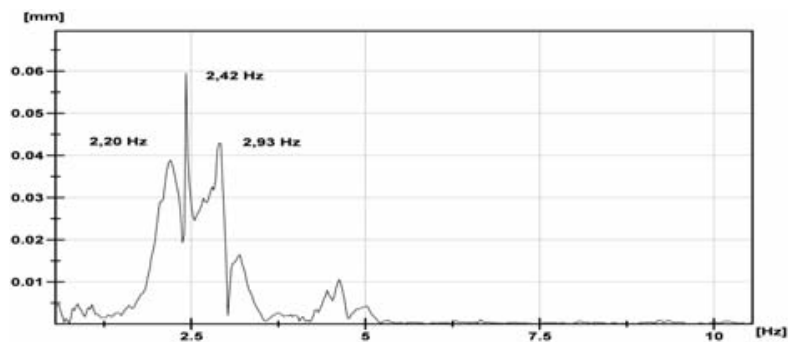

Fig. $7 b$ Frequency composition of the forced vertical displacement $w_{d y n}{ }^{*}(1 / 2, t)$ corresponding Fig. $7 a$.

\subsection{Spectral composition of the vertical vibration of the bridge deck and arches} of:

Vibration properties of the bridge were investigated by means

- A dynamic component for smooth passages $\Delta w_{d y n}{ }^{*}(x, t)$, or passages over the normalised roughness $\Delta w_{d y n}{ }^{*}(x, t)$ at different testing vehicle speeds.

- A dynamic component from impact tests $\Delta w_{d y n}{ }^{* *}(x, t)$ in a characteristic place $(x)$ of the bridge - the impact generated by the back axes of the vehicle from a defined height.

The FFT were computed for each record $\Delta w_{d y n}(x, t)$. The power spectrum curves (PSD) displayed as peak amplitude values $\hat{w}(x)$ were plotted for each passage of the testing vehicle.

The time histories and power spectra of the measured response are shown in Figs. $8 \div 10$ for the bridge deck, and in Fig. $11 \div 12$ for the arches.

\subsubsection{The bridge deck}

Dynamic behaviour of the bridge deck was compared to natural frequencies and mode shapes computed using a linear elastic FEM. Analysed are records from accelerometers directly transformed on displacement $\Delta w_{d y n}(x, t)$ in the vertical direction at the positions $x=\mathrm{A} 1 \div \mathrm{A} 4$ placed at the left girder (L.G.) and the right girder (R.G.) on the bridge deck.

- The bridge deck - Forced vibrations $\Delta w_{d y n}(1 / 2, t)$ generated by the smooth passage of the testing vehicle.

File No. 3.3-A1/A2: Accelerometers A1(L.G. at 1/2), A2(R.G. at $1 / 2$ ) placed on the deck. The vehicle speed $c=25.9 \mathrm{~km} / \mathrm{h}$, the passage time $t_{p}=12.0 \mathrm{~s}$, Figs. $8 \mathrm{a}, \mathrm{b}$.

- The bridge deck - Forced-vibrations $\Delta w_{d y n}(1 / 2, t)$ generated by the smooth passage of the testing vehicle.

File No. 3.3-A3/A4: Accelerometers A3(L.G. at 3/4), A4(R.G. at $3 / 4$ ) placed on the deck. The vehicle speed $c=25.9 \mathrm{~km} / \mathrm{h}$, the passage time $t_{p}=12.0 \mathrm{~s}$, Figs. 9a, b.

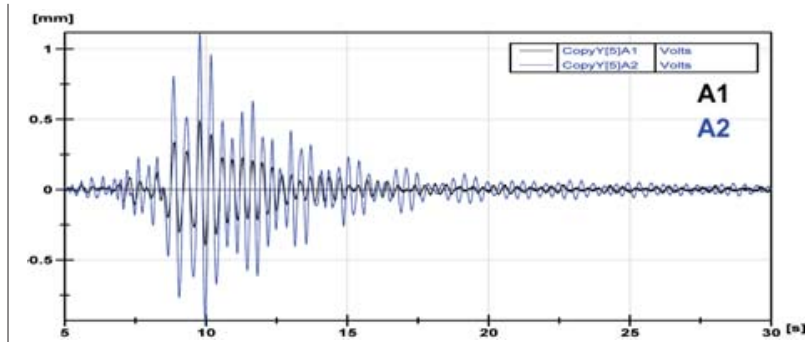

Fig. 8a Time history of the force vertical displacement $\Delta w_{d y n}(1 / 2, t)$

- measured by accelerometers A1(L.G. at 1/2), A2(R.G. at 1/2) on the bridge deck

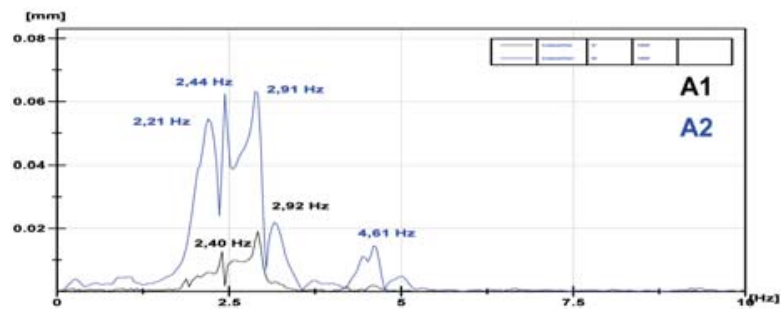

Fig. $8 b$ Frequency composition of the forced vertical displacement - PSD $\Delta w_{d y n}(1 / 2, t)$ corresponding Fig. $8 a$.

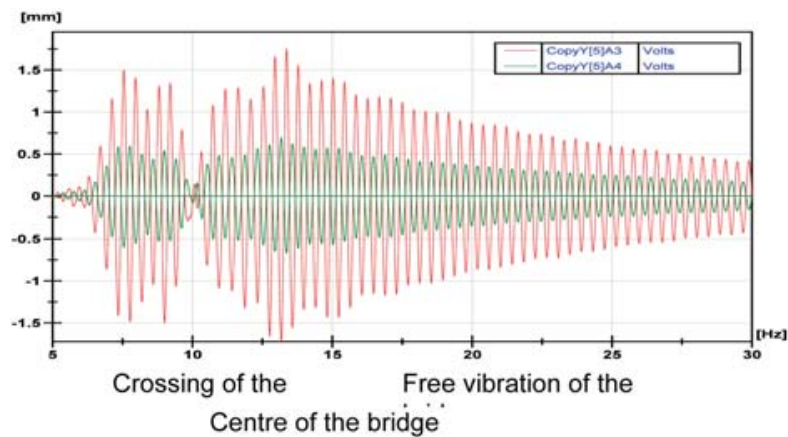

Fig. 9a. Time history of the force vertical displacement $\Delta w_{d y n}(3 / 4, t)$ - measured by accelerometers A3(L.G. at 3/4), A4(R.G. at 3/4) on the bridge deck.

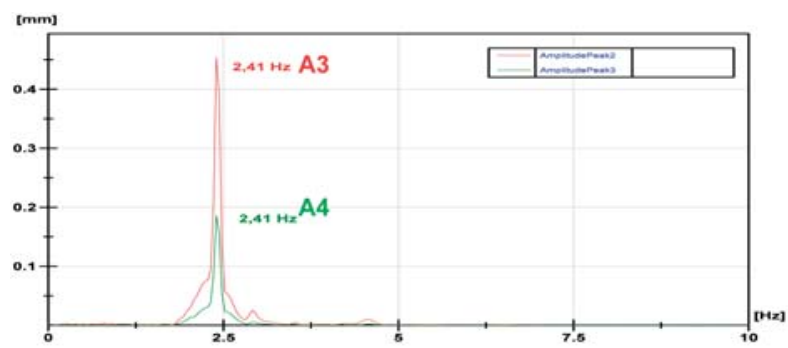

Fig. $9 b$ Frequency composition of the forced vertical displacement PSD $\Delta w_{d y n}(3 / 4, t)$ corresponding Fig. $9 a$.

- The bridge deck - Forced vibrations $\Delta w_{d y n}{ }^{*}(1 / 2, t)$ generated by the passage over the normalised roughness. 
File No. 4.3-A1/A2: Accelerometers A1(L.G. at 1/2), A2(R.G at $1 / 2$ ) placed on the deck. The vehicle speed $c_{p}=25.9 \mathrm{~km} / \mathrm{h}$, the passage time $t_{p}=12.0 \mathrm{~s}$, Figs. 10a, b.

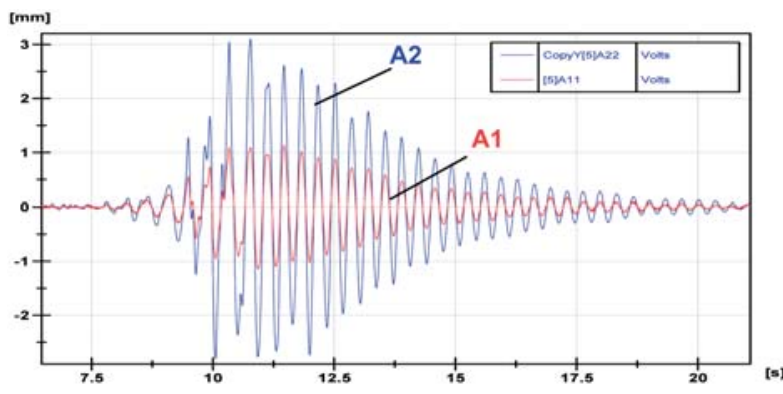

Fig. 10a Time history of the force vertical displacement $\Delta w_{d y n}{ }^{*}(1 / 2, t)$ - measured by accelerometers A1(L.G. at 1/2), A2(R.G. at 1/2) on the deck.

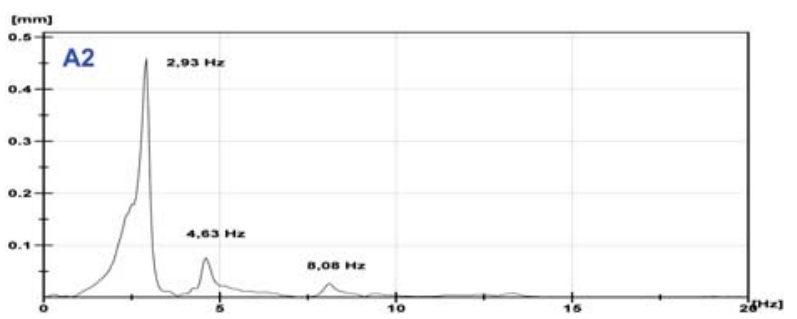

Fig. $10 b$ Frequency composition of the forced vertical displacement - PSD $\Delta w_{d y n}{ }^{*}(1 / 2, t)$ corresponding Fig. 10a.

- The bridge deck - Forced vibrations $\Delta w_{d y n}{ }^{* *}(1 / 2, t)$ generated by the impact of the back vehicle axes on the bridge at $1 / 2$ of the span.

File No. 7.1-A1: Accelerometers A1(L.G. at 1/2) placed on the deck, Figs. 11a, b.

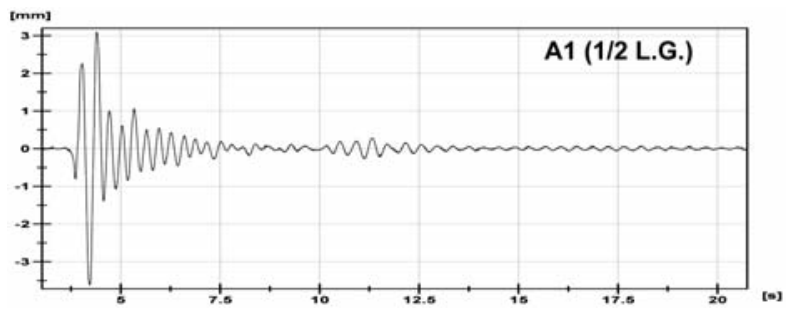

Fig. 11a Time history of the force vertical displacement $\Delta w_{d y n}{ }^{* *}(1 / 2, t)$ generated by the impact - measured by accelerometers A1(L.G. at 1/2) on the deck.

The power spectra shown in Figs. $7 \div 10$ are typical results for the measured response the bridge deck. Forced bridge vibration frequencies for all testing vehicle passages over the bridge preserved

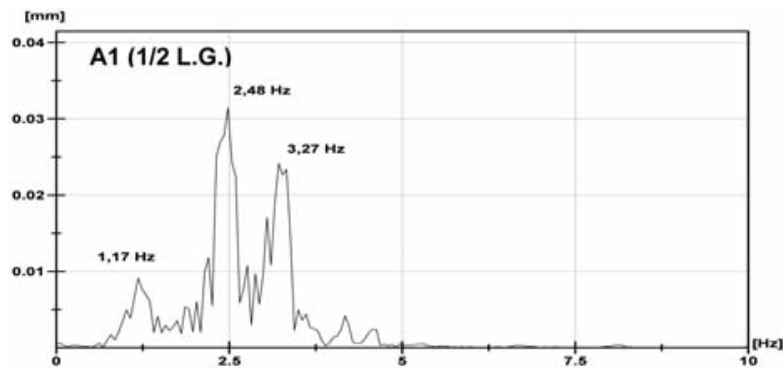

Fig. $11 \mathrm{~b}$ Frequency composition of the forced vertical displacement - PSD $\Delta w_{d y n}{ }^{* * *}(1 / 2, t)$ corresponding Fig. 11a.

nearly the same composition of the vertical vibration of the bridge deck.

- For the passage speeds $c_{p} \approx 20 \div 30 \mathrm{~km} / \mathrm{h}$ the bridge deck vibrates nearly the simple vibration shape with the bearing frequency $f^{*}=2.41 \mathrm{~Hz}$ (see Fig. 8b) which corresponds to the 8th natural frequency $f_{(8)}=2.403 \mathrm{~Hz}$ and the 8th shape of the bridge vibration which is loaded by the vehicle located in the centre of the bridge.

- For the speed $c_{p}>20 \div 30 \mathrm{~km} / \mathrm{h}$ the bridge vibrates in a multiple shape. There are also present natural frequencies, but in the superposition with other frequency components $\left(f_{i}=2.41\right.$; $3.52 ; 3.21 ; 4.03 \mathrm{~Hz}$ ). The frequency composition of vibration is composite and occurs in the range $f^{*}=2.6 \div 5 \mathrm{~Hz}$.

\subsubsection{The bridge arches}

Dynamic behaviour of the bridge arches was also compared to natural frequencies and mode shapes computed using a linear elastic FEM. Analysed records from accelerometers are directly transformed on displacement in the vertical direction ${ }^{(\text {arch })} \Delta w_{\text {dyn }}(x, t)$ and in the horizontal direction ${ }^{(\text {arch })} \Delta u_{d y n}(x, t)$, at the positions $x(\mathrm{~A} 5, \mathrm{~A} 6)$ placed at the top of the left arch (L.A.) and the top of the right arch (R.A.) The time histories and power spectra of the measured arch response are shown in Figs. 12 $\div 13$.

- The arches - Forced-vibrations ${ }^{(a r c h)} \Delta w_{d y n}{ }^{*}(1 / 2, t)$ generated by the passage over the normalised roughness.

File No. 4.3-A5V: The vertical accelerometer $A 5_{V}$ (L.A. at $1 / 2$ ) placed on the arch. The vehicle speed $c=25.9 \mathrm{~km} / \mathrm{h}$, the passage time $t_{p}=12.0$ s, Figs. $12 \mathrm{a}, \mathrm{b}$.

File No. 4.3-A5H: The horizontal accelerometer $A 5_{H}$ (L.A. at $1 / 2)$ on the arch. The vehicle speed $c_{p}=25.9 \mathrm{~km} / \mathrm{h}$, the passage time $t_{p}=12.0$ s, Figs. $13 \mathrm{a}, \mathrm{b}$.

The analyses carried out in the time and frequency domain show and confirm that the wind effect on the vibration of bridge arches was dominant in the time of the measurement of bridge and dominated over the vehicle passages effects, see Fig. 12 and 


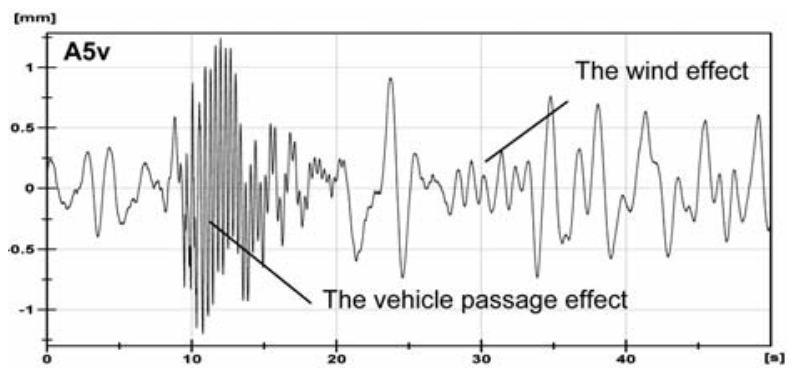

Fig. 12a Time history of the force vertical displacement ${ }^{\text {(arch) }} \Delta w_{\text {dyn }}{ }^{*}(1 / 2, t)$ - measured by accelerometers $A 5_{V}($ L.A. at $1 / 2)$ on the arch.

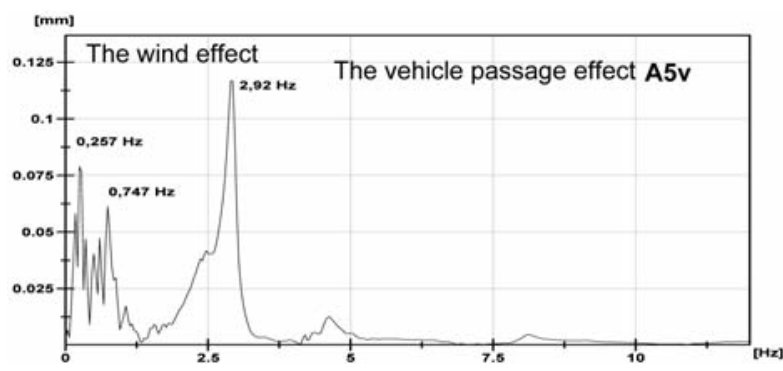

Fig. $12 b$ Frequency composition of the forced vertical displacement - PSD ${ }^{(\text {arch })} \Delta w_{d y n}{ }^{*}(1 / 2, t)$ corresponding Fig. 12a.

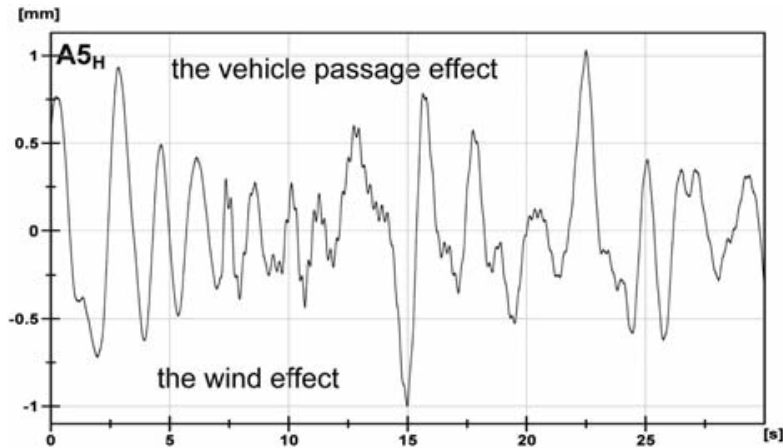

Fig. 13a Time history of the force horizontal displacement ${ }^{(\text {arch })} \Delta w_{d y n}{ }^{*}(1 / 2, t)$ - the accelerometer $A 5_{H}($ L.A. at $1 / 2)$ on the arch.

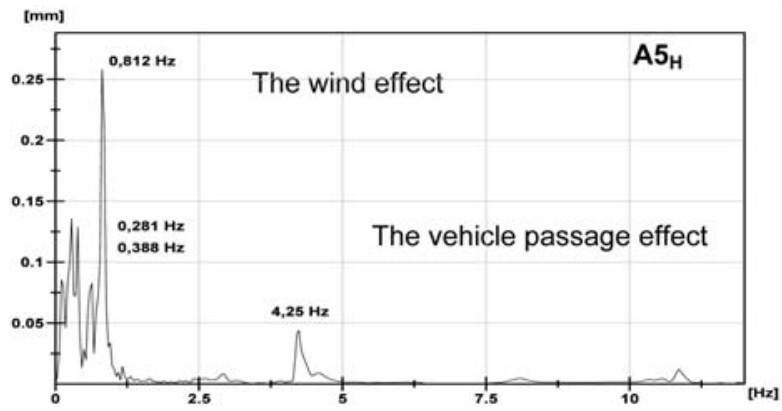

Fig. $13 \mathrm{~b}$ Frequency composition of the forced horizontal displacement corresponding to $13 a$.
Fig. 13. The carrier frequencies of vertical and horizontal vibrations were different:

- The dominant frequencies for the wind effect in the vertical and the horizontal direction lie in the frequency range $f_{\text {wind }} \approx 0.8 \mathrm{~Hz}$.

- The dominant frequencies for the vehicle passage effect lie in the frequency range $f \approx 2.4 \div 4.4 \mathrm{~Hz}$

\section{Conclusions}

The results derived from the dynamic test of the bridge and their comparison with those theoretically predicted permit the assessment of the actual bridge stiffness. They served also to assess the actual structural behaviour in the vertical and horizontal plane. Produced loading tests:

1. Passages of a testing vehicle over the bridge at various speeds as:

- simple smooth passages of the testing vehicle,

- passages over the bridge instrumented by the normalised (6 cm high) roughness.

2. Impact tests on a characteristic place of the bridge - the impact generated by the back axes of the vehicle from a defined height, confirm that the bridge response parameters correspond with the expected ones of the bridge model and the theoretical results. The tests results confirm the expected values of the dynamic coefficients ${ }^{(w)} \delta_{o b s}(x),{ }^{(\varepsilon)} \delta_{o b s}(x)$ and ${ }^{(\Delta w)} \delta_{o b s}(x)$ :

1/ Passages of the testing vehicle Tatra 815 (22t):

- Bridge deck

a/ Direct measurement $\delta_{\text {obs }}(x)$ : $\max { }^{(\varepsilon)} \delta_{\text {obs }}(1 / 2)=1.17$, mean ${ }^{(\varepsilon)} \delta_{\text {obs }}(1 / 2)=1.109 \div 1.16$, $\max ^{(w)} \delta_{\text {obs }}(3 / 4)=1.108$, mean ${ }^{(w)} \delta_{\text {obs }}(3 / 4)=1.06$,

b/ Combined method $\delta_{\text {obs }}(x)$ : ${ }^{(w)} \delta_{\text {obs }}(1 / 2)=1.48$, mean $^{(w)} \delta_{\text {obs }}(3 / 4)=1.17$, ${ }^{(w)} \delta_{o b s}(3 / 4)=1.08$, mean ${ }^{(w)} \delta_{\text {obs }}(3 / 4)=1.06$.

\section{- Bridge arches}

a/ Direct measurement $\delta_{\text {obs }}(x)$ : $\max ^{(\varepsilon)} \delta_{\text {obs }}(1 / 2)^{(O b l)}=1.12$, mean $\delta_{\text {obs }}(1 / 2)=1.11$.

2) Passages of the testing vehicle Tatra 815 (22t) over the bridge instrumented by the normalised $(6 \mathrm{~cm}$ high) roughness:

- Bridge deck: Direct measurement $\delta_{o b s}(x)$ : ${ }^{(w)} \delta_{\text {obs }}(3 / 4)=1.05 \div 1.44$

${ }^{(\varepsilon)} \delta_{\text {obs }}(1 / 2)=1.43 \div 1,69$.

- Bridge arches: Direct measurement $\delta_{\text {obs }}(x)$ : ${ }^{(\varepsilon)} \delta_{\text {obs }}(1 / 2)^{(A r c h)}=1.12$, mean ${ }^{(\varepsilon)} \delta_{o b s}=1.11$.

The dynamic coefficient $\delta_{\text {obs }}(x)$ in characteristic locations $(x)$ of the bridge structure for all measured passages of the testing vehicle fulfil requirements of the Slovak National Standard STN 736209 "Loading tests of bridges" [1], that specifies conditions for the performance of dynamic tests for highway bridges:

$$
\left(\delta_{o b s e r}-1\right) \eta_{d y n} \leq \delta-1
$$




\section{COMMNICOIIONS}

where:

$\delta \quad$ is the dynamic coefficient considered in the design, $\delta=1.172$ $\eta_{d y n}$ is the dynamic efficiency of the applied loading $\eta_{d y n}=0.117$

All applied tests - passages of the testing vehicle and the impact tests show an elastic behaviour of the structure. Measured dynamic displacements and strains achieve values expected from the static calculation. The bridge structure behaved elastically - all measured deformations reached expected small values and no nonlinear behaviour was indicated.

\section{References}

[1] STN 736209 Loading tests of bridges (in Slovak), 1979, in Slovak.

[2] HSP s.r.o.: Technical report (in Slovak) - Objekt 201-00: Background Papers for Loading Test, Brno 2010.

[3] National Instrument: DIAdem Guide for Storing, managing and Analyzing of Measurement Data, Digital, http://www.ni.com/manuals.nsf. 